\title{
Langerhans cell histiocytosis of the atlas in a female adult
}

\author{
Liu Zhiyu, Xue Yajun, Lou Meiqing, Zhao Yaodong
}

Department of Neurosurgery, Shanghai $10^{\text {th }}$ People's Hospital, Tongji University School of Medicine, Shanghai, PR China

Contemp Oncol (Pozn) 2014; 18 (4): 290-291 DOI: $10.5114 /$ wo. 2014.44629

Langerhans cell histiocytosis (LCH) is an infrequent disorder complex. It is a disease of myeloid dendritic cells, lymphocytes, and macrophages mixed with eosinophils and neutrophils [1]. The accumulation of these cells causes the classic lytic bone lesions, skin rashes, lymphadenopathy, and organ dysfunction. Langerhaus cell histocytosis occurs at all ages from infancy up to senility with the peak incidence between 2 and 4 years of age. The male/female ratio is about $3.7: 1$ [2]. We have recently treated a case of $\mathrm{LCH}$ of the atlas in a female adult, which may be the first case of atlas LCH in a female adult reported in the English literature. A 26-year-old woman complained of headache for 10 years, with aggravation for 3 months. No neurological deficits were observed, and no significant medical history or family history was reported. Physical examination demonstrated obvious restriction of the neck motion without neurological deficit or torticollis.

Computed tomography (CT) examination confirmed a sharply osteolytic lesion of the right posterior lacerate foramen area. The atlas right lateral mass, anterior arch and
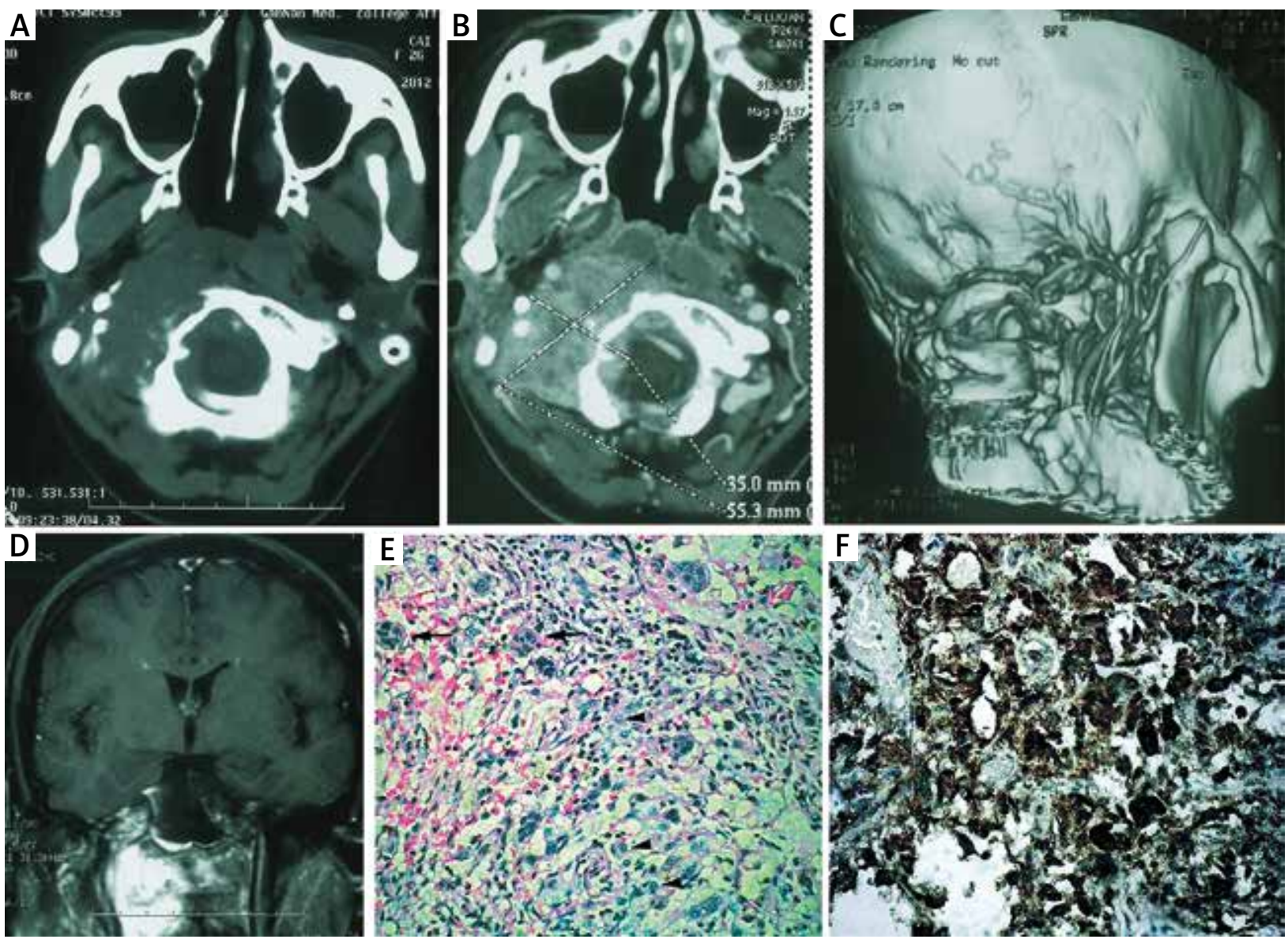

Fig. 1. A) CT scan shows an osteolytic lesion of the right posterior lacerate foramen area, and the lesion's radiological density was similar to muscles nearby; B) after enhancement, the tumor density overall increased obviously, but not very uniformly; C) shows the enlarged gap between the atlas and dentate, and blood vessels of the cervical part were pushed outside; D) for MRI scan, the tumor enhances homogeneously after contrast administration; E) pathological detection, H\&E staining (400x): arrowheads showed Langerhans cell with indented and folded or grooved nuclei resembling coffee beans; arrows show multinuclear giant cells; F) (400x) the main cells are positive for S-100 protein. 
squamous part of occipital bone had been eroded with attenuation. The gap between the atlas and dentate was enlarged. The tumor mass had a similar density as soft tissue such as muscles in a plain CT scan; however, after enhancement, the tumor mass density overall increased obviously, but not very uniformly. On magnetic resonsnce imaging MRI, the soft tissue mass was isointense on T1, but it enhanced homogeneously after contrast administration (Fig. 1A-D). The patient underwent surgical treatment. Under general anesthesia a grey-red tumor of approximately $3.5 \times 5.5 \times 6 \mathrm{~cm}$ was completely removed from the atlas. The tumor had a tough texture with clear border and abundant blood supply.

Histopathologically, it was proven to be LCH. At higher magnification, the proliferating cells had indented and folded or grooved nuclei resembling coffee beans. Multinuclear giant cells were frequently seen. Eosinophils and neutrophils were admixed with the proliferating cells. The main cells were positive for S-100 protein (Fig. 1E and F).

Skull and mandible are common regions of $\mathrm{LCH}$ involvement, and there have been very few published cases describing Langerhans cell histiocytosis of the atlas. On radiological examination, sharply demarcated punch-out osteolytic lesions in bones are characteristic. Final LCH diagnosis is possible based only on histopathology. They are usually 10-15 $\mathrm{mm}$ in size with characteristically folded or multilobular nuclei with typical grooves. Langerhans cells are mixed with inflammatory cells, predominantly acidophilic granulocytes. A reliable immunological marker is S-100 protein, while CD1 $\alpha$ is usually positive too.

Treatment options include surgical excision and curettage of isolated or limited bone disease. External beam radiation with local doses ranging from 2.5 Gy to 120 Gy (usually 5-20 Gy) has also been used. Chemotherapy has been used with good success for disseminated or recalcitrant disease. Overall prognosis is good with survival rates greater than $90 \%$ in patients having limited organ involvement [3]. In our case, the delitescence of the course of disease was more than 10 years, and it showed a slow process of growth of $\mathrm{LCH}$ and a benign nature. After one year of follow-up, there is no evidence of recurrence.

The authors declare no conflict of interest.

The current research was supported by the National Natural Science Foundation of China (No. 81101909), and the Doctoral Program of Higher Education (Specialized Research Fund) of China under Grant No. 20110072120055.

\section{References}

1. Allen CE, Li L, Peters TL, et al. Cell-specific gene expression in Langerhans cell histiocytosis lesions reveals a distinct profile compared with epidermal Langerhans cells. J Immunol 2010; 184: 4557-67.

2. Muramatsu T, Hall GL, Hashimoto S, Miyauchi J, Shimono M. Clinico-pathologic conference: case 4. Langerhans cell histiocytosis (LCH). Head Neck Pathol 2010; 4: 343-6.

3. Howarth DM, Gilchrist GS, Mullan BP, et al. Langerhans'cell histiocytosis: Diagnosis, natural history, management, and outcome. Cancer 1999; 85: 2278-90.

\section{Address for correspondence}

\section{Dr. Zhao Yao-dong}

Department of Neurosurgery

Shanghai $10^{\text {th }}$ People's Hospital

Tongji University School of Medicine

No. 301 Yanchangzhong Road

Shanghai 200072, PR China

tel. 86-21-66306594

fax 86-21-66307359

e-mail: zhaoyd@aliyun.com

Submitted: 8.01 .2014

Accepted: 27.02 .2014 